\title{
ARMONIZAR LOS DERECHOS COOPERATIVOS. ¡REALIDAD! ¿NECESIDAD? ¡TRAMPA!
}

\author{
Hagen Henrÿ \\ Visiting scholar \\ University of Helsinki
}

\section{RESUMEN}

Después de haber advertido al lector de la ambigüedad y de la amplitud de la noción de "armonización", el artículo aborda dos temas: las armonizaciones regionales de leyes de cooperativas y la cuestión si hay una obligación jurídica de armonizar los derechos cooperativos. El artículo agrupa las armonizaciones regionales en categorías con sus diferentes efectos y concluye en la importancia de que esas leyes uniformes se refieren de una u otra manera a los principios cooperativos de la Alianza Cooperativa Internacional, reconocidos universalmente, incluso a través de su integración en la Recomendación sobre la promoción de las cooperativas (No. 193) de la Organización internacional de trabajo en 2002 (R. 193 de la OIT). En cuanto a la cuestión si hay una obligación jurídica de armonizar los derechos cooperativos, el artículo argumenta que sí, la hay; la establece el Párrafo 10 de la R. 193 de la OIT. Al estipular que "los Estados Miembros deberían adoptar una legislación y una reglamentación específicas en materia de cooperativas, inspiradas en los valores y principios cooperativos..." el Párrafo 10 parece contradecir el Párrafo 18 que estipula que "La cooperación internacional debería ser facilitada mediante... el desarrollo a nivel internacional y regional de... leyes comunes." El artículo resuelve esa aparente contradicción en favor del Párrafo 10 porque la traducción de los principios cooperativos en reglas y prácticas jurídicas permite diversidad, lo que "leyes comunes" no hacen, y porque la diversidad es fuente del desarrollo sostenible. El artículo concluye al discutir algunos problemas de esa traducción.

PALABRAS CLAVE: Armonización del derecho, principios cooperativos, derecho cooperativo, Recomendación sobre la promoción de las cooperativas (No. 193) de la Organización internacional del trabajo.

CLAVES ECONLIT / ECONLIT DESCRIPTORS: K19, K33, P13.

Cómo citar este artículo/How to cite this article: HENRŸ, H.: "Armonizar los derechos cooperativos. ¡Realidad! ¿necesidad? ¡trampa!", CIRIEC-España, Revista Jurídica de Economía Social y Cooperativa, $\mathrm{n}^{\circ} 39,2021$, pp. 45-66. DOI: 10.7203/CIRIEC-JUR.39.21516 


\section{HARMONIZING COOPERATIVE LAWS. REALITY! NECESSARY? TRAP!}

\section{EXPANDED ABSTRACT}

After having underlined at the beginning the polysemy of the word "harmonization" in the context of the approximation of laws, the article pursues two objectives: The first is to present cases of regional harmonizations of cooperative laws. The second is to demonstrate that the 2002 Promotion of Cooperatives Recommendation (No. 193) of the International Labor Organization, despite a somewhat unclear wording to the contrary in several of its paragraphs, suggests that the cooperative laws should not be unified, but rather that the cooperative principles, as enshrined in the 1995 International Cooperative Alliance Statement on the cooperative identity, be translated into legal rules and praxes.

The article recognizes that its objectives make for its rather limited scientific value. It excludes more than it includes. Firstly, it does not consider intra-national harmonizations, of which there is a growing number, given the dissatisfaction with either the multitude of sectoral cooperative laws in a country (examples were, are or might be France, Greece, Japan, Kazakhstan, South Korea) or the multitude of cooperative laws due to the constitutional order of a country (examples were or are Australia, Canada, Russian Federation, Spain, United States of America). The article also disregards harmonizations of cooperative laws effectuated by the harmonization of other laws than that of the laws on cooperatives, such as for example, tax laws, accounting standards, competition law. The article does not deal with the different motivations behind the harmonizations, which are mostly political or economic, nor does it consider cases of unintended harmonizations, such as the isomorphization of all enterprise forms by law. Secondly, the article does not differentiate between the modes and grades of harmonization. Indeed, the word "harmonization" covers such different issues as the unification of laws resulting in uniform laws and the informal coordination of cooperative law-making, as well as everything in between.

As for its first objective, the article classifies regional cooperative laws into those which are directly applicable in several countries; those which require transforming into national law before they may be applied; those which regulate cross-border cooperatives, but do not regulate all necessary matters, and which are either directly applicable or require transformation into national law; and regional laws which are currently being elaborated.

The article mentions for the first category the 2010 Uniform cooperative act of OHADA, the Organization for the harmonization in Africa of business law and the 2014 East African Community (uniform) Cooperative Societies Bill. The first one applies directly since 2011 in 
(pp. 45-66)

the 17 Member states of OHADA, the second one is not yet in force. In the second category we find the 1997 Model law on cooperatives and their unions of the Commonwealth of Independent States (CIS) and the 2008 Ley marco para las cooperativas de América Latina, the Framework Law for cooperatives in Latin America. The first one is not a binding law, but the CIS suggests to its Member states to follow this model. The second one is also a model law. However, it was adopted by a non-governmental organization, the regional organization for the Americas of the ICA. Its legal value stems from the fact that the Parlatino, the Latin American Parliament, endorsed its content with insignificant changes. In the third category we find the European Union Council Regulation (EC) No. 1435/2003 on the Statute for a European Cooperative Society (SCE) and the 2009 Estatuto de las cooperativas del Mercosur. These texts regulate cross-border cooperatives. As they are incomplete, the national laws applicable to cooperatives in the country where they are registered apply in addition. The EU Regulation came into force in 2006; the Estatuto Mercosur will come into force once transformed by all Member states of Mercosur into national law. As far as the fourth category is concerned, the African Union is elaborating a model cooperative law for its Member states and the Pacific Island countries are contemplating the harmonization of their cooperative laws.

The effects of these harmonizations are mixed. Besides the usual diversification through implementation, all of them have their specific outcomes. The most far-reaching concern the two African uniform laws. In many instances the OHADA Uniform act is not even known in the countries concerned; some of the Member states of the EAC, whilst not ratifying and/ or transforming the 2014 Uniform act, engage in national cooperative law making and do not wait for the outcome of the initiative of the $\mathrm{AU}$ to have a model cooperative law for its Member states. As concerns the EU Regulation, it produced harmonizing effects on some cooperative laws of EU Member states despite the explicit aim of the EU Commission to not harmonize the national laws. Whatever the effects are in terms of harmonization, intended or unintended, all of the mentioned harmonizations refer in one way or the other to the cooperative principles. The question is whether there is a farther-reaching legal obligation to translate the cooperative principles into legal rules and praxes.

The second part of the article deals with this question. It starts by summarizing and categorizing the different ways of how a growing number of regional and national cooperative laws refer to the cooperative principles. This categorization is to help find answers to the question of what the effects of these references are. Without discussing this point any further the article takes these references as a point of fact to then deal with the question of whether Paragraph 10. of the ILO R. 193 creates a legal obligation to translate the cooperative principles into legal rules and praxes. The Paragraph states that the Member states [of the IL0] should "adopt specific legislation and regulations on cooperatives, which are guided by the cooperative values and principles...". The article summarily presents arguments in favor of 
such an obligation and then discusses its content. It does so by interpreting the wording of Paragraph 10 as compared to that of Paragraph 18. Paragraph 18 states that "International cooperation should be facilitated through "developing ... common regional and international guidelines and legislation". At first sight, Paragraph 10 contradicts Paragraph 18. But it must be read in conjunction with the legal concept of sustainable development. This concept requires diversity. The translation of principles into legal rules and praxes is more likely to maintain diversity than "common legislation". While the consensus on this is growing, the conclusion raises a number of additional questions. For example: To whose cooperative principles does the ILO R. 193 refer to, to those of the ICA, to its own or to more general ones? How to translate these principles into law?

KEYWORDS: Harmonization of laws, cooperative principles, cooperative law, Promotion of Cooperatives Recommendation No. 193 of the International Labor Organization. 


\section{SUMARIO}

I. Introducción. II. Leyes de cooperativas regionales y sus efectos. 1. Leyes regionales. 2. Efectos. III. La armonización de los derechos cooperativos por el reconocimiento de los principios cooperativos. 1. La armonización de los derechos cooperativos por el reconocimiento voluntario de los principios cooperativos. 2. La armonización obligatoria de los derechos cooperativos según el Párrafo 10. (1) de la Recomendación No. 193 de la Organización Internacional de Trabajo sobre la promoción de las cooperativas. IV. Conclusión. Bibliografía.

\section{Il mio supplizio \\ è quando \\ non mi credo \\ in armonia}

Giuseppe Ungaretti, I fiumi, en: Die Heiterkeit / L'Allegria

La loi, en général, est la raison humaine en tant qu'elle gouverne tous les peuples de la terre; et les lois politiques et civiles de chaque nation ne doivent être que les cas particuliers où s'applique cette raison humaine.

Elles doivent être tellement propres au peuple pour lequel elles sont faites que c'est un très grand hasard si celles d'une nation peuvent convenir à une autre. Montesquieu, De l'esprit des lois, Première Partie, I, 3 (Des lois positives)

\section{Introducción}

Desde hace milenios políticos, economistas y comparatistas del derecho creen compadecerse de Ungaretti. Cualquier diferencia entre derechos les incómoda a ellos. Impulsan su armonización donde pueden. Al otro lado, Montesquieu y sus seguidores ${ }^{1}$ no se compadecen de él. Para ellos, no vale lamentar lo que no se puede cambiar: apenas armonizados, los derechos se diversifican a través de sus interpretaciones-aplicaciones. Estas variarán según las culturas subyacentes en los derechos armonizados. Pero, la diversidad es inherente a la naturaleza del derecho. El derecho armoniza los necesarios diversos. Si la mencionada diversificación a través de las interpretaciones-aplicaciones se opusiera a la armonización, el derecho sería imposible.

1. Por ejemplo, el comparatista Pierre Legrand. Entre otros de sus artículos, vid. LEGRAND, P.: "The Impossibility of Legal Transplants", Maastricht Journal of European and Comparative Law, no 4(2), 1997, pp. 111-124. 
En un mundo en donde nos conectamos cada vez más con desconocidos necesitamos más que nunca el derecho porque nos permite vivir en armonía: "[Le] droit existe pour empêcher le monde de se détruire"2.

Así, el suplicio de Ungaretti adquiere su pleno sentido: él teme la unisonancia. Lo que parece a una paradoja, en realidad es la cuerda floja entre armonía y unisonancia, entre multiformidad y uniformidad, en que andamos. Debemos uniformar cuando sea necesario sin, por ello, comprometer la posibilidad de diversidad.

$\mathrm{Al}$ armonizar los derechos cooperativos no solo corremos el riesgo de restringir esta posibilidad, sino también él de violar el concepto jurídico de desarrollo sostenible reconocido por la Corte Internacional de Justicia ${ }^{3}$, un concepto que se integra siempre más en todas las áreas del derecho.

El objetivo de ese artículo es doble: Primero, presentar casos de leyes organizativas de cooperativas regionales; y, segundo, demostrar a través de la interpretación de la Recomendación No. 193 sobre la Promoción de las Cooperativas de la Organización Internacional del Trabajo del ańo 2002 (R. 193 de la OIT) ${ }^{4}$ que lo que el derecho público internacional exige no es la uniformización de las reglas jurídicas aplicables a las cooperativas, sino el reconocimiento de los principios cooperativos universales contenidas en la Declaración sobre la Identidad Cooperativa de la Alianza Cooperativa Internacional de 1995 (principios cooperativos) ${ }^{5}$ y su traducción en reglas y prácticas jurídicas que reflejen la necesaria diversidad de los mundos ${ }^{6}$.

2. Vid. CARBOnNIER, J.: "Le doit au non-droit". En: Paul Ricour, Jacques Ellul, Jean Carbonnier, Pierre Chaunu: Dialogues (aut. ABEL, O.), Labor et Fides, Ginebra, 2012, p. 76.

3. Vid. CORTE INTERNACIONAL DE JUSTÍCIA [CIJ]: Reports of judgments, advisory opinions and orders. Case concerning: The Gabcikovo-Nagymaros Project (Hungary/Slovakia), Corte Internacional de Justicia (CIJ), La Haya, 1997, párrafo 140.

Para la historia del concepto, vid. BEKHECHI, M.A.: "Quelques notes et réflexions sur le statut du droit international du développement durable". En: Variations sur le système international. Mélanges offerts en l'honneur du Professeur Mohamed Lamouri (dir. ESSAID, M.J.), Najah Al Jadida, Casablanca, 2010, pp. 107137; GEHNE, K.: Nachhaltige Entwicklung als Rechtsprinzip, Mohr Siebeck, Tübingen, 2011; y HENRŸ, H.: "Sustainable Development and Cooperative Law: Corporate Social Responsibility or Cooperative Social Responsibility?", International and Comparative Corporate Law Journal (ICCLJ), no 10(3), 2013, pp. 58-75.

4. Vid. INTERNATIONAL LABOUR ORGANIZATION [ILO]: Promotion of Cooperatives Recommendation, $\mathrm{n}^{\circ}$ 193, International Labour Organization (ILO), Ginebra, 2002.

5. Vid. International Co-operative Review, no 88(4), 1995, 85 s. y http://ica.coop/en/cooperatives/cooperativeidentity.

6. Esa duplicación de diversidad es para subrayar la vital importancia de la diversidad en sus dos aspectos, la diversidad biológica y la diversidad cultural en todas sus expresiones, inclusive una diversidad de formas de empresa. 
El enfoque del artículo en las leyes regionales sigue el uso del término "armonización de derechos". En general, se entiende por "armonización de derechos" la reducción del número de diferentes textos de leyes nacionales aplicables a una misma materia. Este enfoque indica el valor científico limitado del artículo en un doble sentido: Primero, el artículo no considera los casos de armonizaciones intra-nacionales ${ }^{7}$; no considera la armonización de los derechos cooperativos por la armonización de otras leyes e actos jurídicos que en su conjunto con las leyes de cooperativas constituyen el derecho cooperativo ${ }^{8}$; no discute los diferentes motivos de las armonizaciones ${ }^{9}$; no trata de casos de armonizaciones que, contrariamente a los casos presentados aquí, no son el resultado de una armonización intencionada o explícita ${ }^{10}$, sino el resultado

La expresión "diversidad de los mundos" es la traducción del título del primer número de la revista "Cahiers de l'Institut d'Etudes du Développement" de la Universidad de Ginebra. El título era "La pluralité des mondes. Théories et pratiques du développement”. El título era programático para la revista.

7. Por razones diversas: (iniciativas) en Australia, Canadá, Espańa, Estados Unidos de América, Francia, Grecia, Japón, Kazakstán. Ver también los cambios de la Constitución de India en 2021.

A mencionar que en algunos casos un país decide adoptar varias leyes de cooperativas después de un periodo con una sola ley. El más reciente ejemplo es la Grecia. Al revés, hay al menos un país, el Portugal, que tiene un código cooperativo que regula todas las cuestiones relativas a las cooperativas en un solo corpus de ley, el Código Cooperativo (Lei 119/2015, en su versión de la Lei 66/2017), es decir cuestiones relacionados con la noción amplia del derecho cooperativo, tal como presentada en la nota en pie de página 8.

8. En general, se entiende por "derecho cooperativo" todas aquellas normas jurídicas - leyes, actos administrativos, resoluciones judiciales, jurisprudencia, reglamentos y estatutos cooperativos y cualquier otra fuente de derecho - que regulan la estructura y las operaciones de las cooperativas como empresas en el sentido económico y como entidades en el sentido jurídico.

Esa noción del derecho cooperativo refleja un concepto amplio, que no sólo comprende la legislación cooperativa propiamente dicha (ley de cooperativas), sino también todas las normas jurídicas adicionales que dan forma a esta institución y regulan sus operaciones. Entre las normas de este tipo presentes en cualquier sistema jurídico deben mencionarse: la constitución, el derecho laboral, la legislación sobre competencia, el derecho tributario, los estándares internacionales sobre contabilidad y normas prudenciales, las normas sobre registros contables, sobre la protección del consumidor, sobre auditoría y quiebra. Debe complementarse con la consideración de reglas y prácticas de implementación, por ejemplo: los mecanismos prudenciales y los procedimientos y sistemas de auditoría y registro, así como las prácticas de los oficiales quienes implementan los reglamentos gubernamentales (en ese sentido, vid. GALLIGAN, D.J.: Law in Modern Society, Oxford University Press, Oxford, 2007, pp. 293 y ss.). Incluye también los procedimientos y mecanismos jurisdiccionales y legislativos y la política jurídica. Vid. HENRŸ, H.: Orientaciones para la Legislación Cooperativa, Organización Internacional del Trabajo (OIT), Ginebra, 2013, Recuadro 2.

9. Pueden ser políticos y/o económicos.

10. El modo explícito de armonización de derechos, sobre todo la de leyes, es un fenómeno estrechamente ligado a la formación de los imperios -romano, coloniales y otros- y sobre todo la formación de los estados nacionales después y como consecuencia del Tratado Westfaliano, cuya segunda edición era la formación de los estados pos-coloniales. Estas formaciones siguen la lógica de consolidación de espacios políticos. Hoy en día la armonización explícita de leyes obedece a otra lógica, una lógica económica. En la medida que lo eco- 


\section{de una armonización espontánea, no intencionada o implícita ${ }^{11}$. A mencionar en este último contexto las armonizaciones que se operan entre leyes organizativas apli- cables a diferentes tipos de empresas ${ }^{12}$. El artículo no trata tampoco de la cuestión si}

nómico se globaliza, la unidad del político y del económico se disuelve y se acompaña con frecuencia de una armonización de los derechos (a veces según criterios exigidos o elaborados por actores privados). En lo del derecho cooperativo mencionamos la Ley marco para las cooperativas de América Latina de la ACI (región Américas), las Leyes de cooperativas uniformes de la OHADA y de la East African Community EAC (región de África), así como los estándares del International Accounting Standards Board (IASB) y del International Financial Reporting Board (IFRB), así como las reglas de Basilea. Tienen como motivo él de consolidar espacios económicos más grandes que los territorios nacionales. Ese fenómeno lo describió ya Koizumi (vid. KOIZUMI, T.: "Cultural Diffusion, Economic Integration and the Sovereignty of the Nation-State", Rechtstheorie. Beihefte (BH RT), no 12, 1991, pp. 313 y ss.).

11. El modo implícito coincide con la historia del derecho comparado. Para una breve historia del derecho comparado, vid. GOLTZBERG, S.: Le droit comparé, Presses Universitaires de France (PUF), París, 2018. Ignoramos lo que el Rey Hamurabi - considerado el primer comparatista - hizo con los resultados de un estudio de los derechos en otros países. Pero, sí, hay razones para pensar que les utilizó para mejorar el derecho de su reino, armonizando así los derechos de los países de la región. Otro ejemplo de una armonización implícita es la influencia del derecho romano sobre el pensamiento de los juristas al familiarizarse durante siglos con los principios, las figuras y las reglas de este derecho (lo que en general se entiende por Recepción del derecho romano) y a través el uso del latín jurídico en los lenguajes jurídicos de un gran número de países (en ese sentido ver recientemente MATTILA, H.E.S.: El latín jurídico. Historia, uso internacional, problemas de comunicación, Ediciones Jurídicas Olejnik, Santiago de Chile, 2020). Otros ejemplos de armonizaciones implícitas son los Restatements por el American Law Institute, un organismo privado cuyas propuestas las siguen con frecuencia los Estados federales de los Estados Unidos de América. Por fin, no se puede ignorar el efecto armonizador de la cooperación técnica en materia de derecho. En lo del derecho cooperativo la más influente era la basada en la llamada British Indian Pattern of Cooperation, un conjunto de leyes y reglamentos introducidos por el poder colonial británico en la India a principio del siglo 20 y que servía de pauta en un gran número de países, no solo países del imperio británico. Para su historia, vid. MÜNKNER, H.H. (ed.): One Hundred Years: Co-operative Credit Societies Act in India - A Unique Experience of Legal Social Engineering, Centenary Round Table on Indian Co-operative Movement - Retrospect and Prospect, Marburg, 2005. Para un resúmen de la cooperación técnica en materia de derecho cooperativo, vid. INTERNATIONAL LABOUR ORGANIZATION [ILO]: The Story of the ILO's Promotion of Cooperatives Recommendation, 2002 (No. 193): A review of the process of making ILO Recommendation no. 193, its implementation and its impact, International Labour Organization (ILO), Ginebra, 2015.

12. Esta armonización se opera a través de tres fenómenos principalmente: La "mercantilización" de las cooperativas a través del alineamiento de sus características estructurales jurídicas a las de las sociedades mercantiles; la jurídificación de la responsabilidad social de todos los tipos de empresas y la extensión de esa responsabilidad a aspectos societales (Responsabilidad Social y Societal de las Empresas, RSSE) que tiene que ver con la superposición de la noción de stakeholder value a las nociones de share holder value (sociedades a capital) y member value (empresas cooperativas); y la armonización a través de la integración organizativa de las empresas de todo tipo en cadenas de valor globales.

En cuanto al fenómeno de "mercantilización", ver recientemente FAJARDO GARCÍA, I.G.: "El fomento de la sociedad cooperativa mediante una legislación adecuada, 40 ańos después", CIRIEC-España, Revista de Economía Pública, Social y Cooperativa, no extraordinario, diciembre, 2018, pp. 141-159; HENRŸ, H.: “Quo Vadis Cooperative Law?”, CCIJ Report, no 72, 2014, pp. 50-61; y VILLAFAŃEZ PEREZ, I.: "Algunas 
la institución que "armoniza" las leyes tiene la competencia de hacerlo" ${ }^{13}$. Segundo, en consonancia con el uso general e incoherente del término "armonización" el artículo no diferencia entre los diferentes modos y grados de armonización los cuales el término esconde. En efecto, el término recobra fenómenos desde unificaciones hasta coordinaciones informales. Un término más adecuado y genérico sería él de "aproximación" 14 .

Antes de concluir la siguiente Parte II trata pues de leyes de cooperativas regionales y sus efectos; la Parte III trata de la armonización de los derechos cooperativos a través del reconocimiento de los principios cooperativos y su traducción en reglas y prácticas jurídicas.

reflexiones en torno a la necesidad de integrar la perspectiva cooperativa en el estudio y desarrollo del ordenamiento jurídico". En: Co-operative Studies in Education Curricula. New Forms of Learning and Teaching (eds. HENRŸ, H., HYTINKOSKI, P. \& KLÉN, T.), Ruralia Institute of the University of Helsinki, Seinäjoki, 2017, pp. 54-71.

13. Por ejemplo, OHADA, la Organisation pour l'harmonisation en Afrique du droit des affaires, tiene la competencia de armonizar los derechos económicos de sus estados miembros. En realidad, los armoniza en el sentido de unificarlos.

14. En el orden alfabético se usan los siguientes términos: “aproximación" que nos parece el término el más neutro porque indica solo el hecho de un acercamiento de diferentes derechos sin indicar el grado de aproximación, ni su motivo, ni si se trata de armonización implícita o explicita; "armonización", término el más utilizado para significar casi toda la gama de modos de aproximación y por eso el más impreciso; "mercantilización" o "comercialización" (ver nota en pie de página 12); "convergencia" ("convergencia” significa el proceso de uniformación de las estructuras de gobernanza de todas las formas de empresa con el motivo de mejorar la solidez económica de las empresas y así mejorar la seguridad económica); "coordinación" (un modo de aproximación informal practicado por los Estados miembros del Nordic Council, por ejemplo), "copiar", "estandarización " (término utilizado por ejemplo por Willy Tadjudje en su artículo sobre la Ley uniforme de cooperativas de OHADA (vid. TADJUDJE, W.: "Standardization of cooperative law in Africa: A comparative analysis between the OHADA uniform act related to cooperative societies and the East Africa community's co-operative societies bill", International Journal of Cooperative Law (IJCL), no 3, 2020, pp. 31-45); "leyes comunes" (Párrafo 18. d) de la R. 193 de la OIT; "trasplantación” (ver la obra de Watson); "unificación".

Estos términos se encuentran a veces en las denominaciones de organizaciones o agencias (por ejemplo: OHADA, UNCITRAL, Unidroit), a veces designan las atribuciones de ellos (por ejemplo, las de la Unión Europea), a veces se encuentran solo en la literatura. En general, describen procesos más o menos formales cuyos efectos variarán según un gran número de factores, tal como, por ejemplo, la relación entre los derechos nacionales y los derechos regionales e internacionales, la existencia o no de una corte común (caso de la Unión Europea, de OHADA y de la East African Community, por ejemplo) y la formación común o no de los jueces de los estados participantes o miembros. 


\section{Leyes de cooperativas regionales y sus efectos}

\section{Leyes regionales}

A continuación, se presentan ejemplos de leyes de cooperativas regionales (supra-estatales). Las clasificamos en cuatro categorías: Leyes aplicables directamente en varios estados; leyes que necesitan una transformación en los derechos nacionales respectivos; leyes que regulan de manera incompleta cooperativas transfronterizas, aplicables directamente o después de su transformación en derecho nacional; y leyes regionales en estado de elaboración.

Entran en la primera categoría el Acte uniforme relatif aux sociétés coopératives de OHADA, la Organisation pour l'harmonisation en Afrique du droit des affaires, de 2010, y la East African Community (EAC) Cooperative Societies Bill de 2014. En vigor desde 2011, el Acte uniforme de OHADA remplaza las leyes nacionales de los 17 Estados miembros de OHADA ${ }^{15}$, salvo si el Acte prevea una excepción o no regule una materia regulada por el derecho nacional ${ }^{16}$. Una vez en vigor, la East African Community Cooperative Societies Bill se aplicará de la misma manera ${ }^{17}$ en los Estados miembros de la East African Community ${ }^{18}$.

Forman parte de la segunda categoría la Ley modelo relativa a las cooperativas y sus uniones de la Comunidad de los Estados Independientes (CEI) y la Ley marco para las cooperativas de América Latina. La Ley modelo de la CEI, adoptada por la asamblea interparlamentaria de la CEI en $1997^{19}$, no es jurídicamente vinculante, pero la CEI incita a sus Estados miembros de respectarla en vista de una armoniza-

15. Los Estados miembros de OHADA son Benín, Burkina Faso, Camerún, África Central, Comoras, Congo, Côte-d’Ivoire, Gabón, Guinea-Conakry, Guinea-Bissau, Guinea Ecuatorial, Mali, Níger, República democrática del Congo (RDC), Senegal, Chad, Togo.

16. Ver su Artículo 2. Para más detalle, vid. TADJUDJE, W.: Le droit des coopératives et des mutuelles dans l'espace OHADA, Editions Larcier, Bruselas 2015; HIEZ, D. \& TADJUDJE, W.: "The OHADA Cooperative Regulation”. En: International Handbook of Cooperative Law (eds. CRACOGNA, D., FICI, A. \& HENRŸ, H.), Springer, Heidelberg, 2013, pp. 89-113; y HIEZ, D. \& KENMOGNE SIMO, A. (eds.): Le Droit des sociétés coopératives $O H A D A$, Presses Universitaires d'Aix-Marseille Université, Marsella, 2017.

17. Ver su Artículo 54. Para una introducción a esa Ley uniforme, vid. TADJUDJE, W.: "The East African Community's Cooperative Regulation”, International Journal of Cooperative Law (IJCL), $\mathrm{n}^{\circ}$ 1, 2018, pp. 148-166.

18. Los Estados miembros de la East African Community son Burundi, Kenia, Ruanda, Sudan del Sur, Tanzania y Uganda.

19. Los Estados Miembros de la CEI son Azerbaiyán, Armenia, Bielorrusia, Kazakstán, Kirguizistán, Moldavia, Federación de Rusia, Tayikistán, Turkmenistán, Ucrania et Uzbekistán. 
ción de los derechos cooperativos de los Estados miembros ${ }^{20}$. La Ley marco para las cooperativas de América Latina constituye un caso particular. Se adoptó en 2008 por una organización no-gubernamental, la organización regional de la ACI para las Américas. Su valor jurídico lo obtiene por el hecho de que el Parlatino, el parlamento latinoamericano, compuesto de delegados de los parlamentos nacionales de la región, la endosó sin modificaciones relevantes.

Encontramos en la tercera categoría el Reglamento (CE) nº 1435/2003 del Consejo de la Union Europea de 2003 relativo al Estatuto de la sociedad cooperativa europea (Reglamento de la Union Europea), así como el Estatuto de las Cooperativas del Mercosur de $2009^{21}$. Los dos textos regulan cooperativas transfronterizas. Lo hacen de manera incompleta, en el sentido de que los derechos nacionales de los Estados miembros de la Unión Europea y del Mercosur vienen necesariamente completarlos. El Reglamento de la Unión Europea entró en vigor en 2006; el Estatuto del Mercosur entrará en vigor una vez integrado en las leyes nacionales de todos los Estados miembros del Mercosur ${ }^{22}$. Hasta ahora solo el Uruguay lo transformó en derecho nacional ${ }^{23}$.

Mencionamos para la cuarta categoría una iniciativa de la Unión Africana y una en la región de las Islas del Pacífico. La Unión Africana está elaborando una ley modelo que oriente a los legisladores nacionales y regionales ${ }^{24}$ del continente. Según una información por parte de la ACI, las autoridades de la región de las Islas del Pacífico y la organización regional de la ACI para Asia y el Pacífico examinan la necesidad y posibilidad de armonizar las bases de las legislaciones cooperativas de la región ${ }^{25}$.

20. Vid. De LUCA, N.: "Russia". En: International Handbook of Cooperative Law (eds. CRACOGNA, D., FICI, A. \& HENRŸ, H.), Springer, Heidelberg, 2013, pp. 667-685.

21. Para una discusión más detallada de estos dos textos ver, respectivamente, FISCHER, T.: "Das Statut der Europäischen Genossenschaft”. En: Genossenschaftsrecht in Europa (eds. THEURL, T. \& GREVE, R.), Shaker, Aachen, 2001, pp. 167-186; SCHULZE, R. (ed.): Europäische Genossenschaft SCE - Handbuch, Nomos, BadenBaden, 2004; y CRACOGNA, D.: "The Statute of Mercosur Cooperatives". En: International Handbook of Cooperative Law (eds. CRACOGNA, D., FICI, A. \& HENRŸ, H.), Springer, Heidelberg, 2013, pp. 153-164.

22. Los estados miembros del Mercosur son Argentina, Brasil, Paraguay, Uruguay, Venezuela (suspendido).

23. Ver su Ley No 18723 (2010).

24. OHADA y EAC.

25. Información por parte del Legislation Coordinator de la ACI, el Sr. Santosh Kumar, el 4 de febrero de 2020. 


\section{Efectos}

Hay mucha incertidumbre no solo en cuanto a los efectos armonizadores de estas leyes regionales de cooperativas, sino también en cuanto a sus efectos en general. El Acte uniforme de OHADA encuentra problemas de aplicación básicos, tales como su ignorancia en algunos Estados miembros; interpretaciones contradictorias; aplicaciones deficientes ${ }^{26}$. La East African Community Cooperatives Societies Bill todavía no está en vigor. Además, las actividades legislativas de algunos de los Estados miembros de la EAC indican su desinterés por esta ley uniforme. Se desconocen los efectos de la Ley modelo de la CEI. La Ley marco para las cooperativas de América Latina es un punto de referencia en los debates sobre el derecho cooperativo de la región, pero no se puede afirmar algo por cierto a sus efectos armonizadores. Lo interesante es que el Reglamento de la Unión Europea, cuyo motivo no era armonizar los derechos cooperativos de los Estados miembros de la Unión Europea, sino crear un nuevo tipo de cooperativa ${ }^{27}$, tenía un efecto armonizador. Algunos de los Estados miembros reformaban sus leyes cooperativas, introduciendo reglas parecidas a las de las leyes favoritas. El Estatuto del Mercosur todavía no está en vigor. Obviamente, las iniciativas en Africa y en el Pacifico todavía no pueden ser consideradas. Además, todos esos ejemplos no escaparán al fenómeno de diversificación pos-adopción. Parece a una paradoja: El grado de aproximación disminuye con la intensidad técnica de la armonización. Al otro lado, observamos efectos de armonización a pesar de los motivos explícitos contrarios. ¿Es decir que Montesquieu y sus seguidores tienen razón? No es cierto. Pero, lo que nos interesa más que el efecto armonizador, o más precisamente: el efecto unificador, es la referencia en casi todos los textos presentados a los principios cooperativos. La diferencia entre principios jurídicos y reglas jurídicas ${ }^{28}$ permite lo necesario: el reconocimiento y la armonización de la interpretación de los principios sin, por

26. Vid. TADJUDJE, W. (dir.): Le droit des coopératives en Afrique. Réflexions sur l'Acte uniforme OHADA, ÉPURE - Editions et Presses Universitaires de Reims, Reims, 2021.

27. Vid. COMISIÓN DE LAS COMUNIDADES EUROPEAS [CCE]: Communication from the Commission to the Council and the European Parliament, the European Economic and Social Committee and the Committee of Regions on the promotion of co-operative societies in Europe (COM (2004) 0018 final), Comision Europea (CE), Bruselas, 2004. Similar, vid. MIRIBUNG, G.: The Agricultural Cooperative in the Framework of the European Cooperative Society. Discussing and Comparing Issues of Cooperative Governance and Finance in Italy and Austria, Springer, Cham, 2020.

28. Los principios jurídicos pueden justificar varias decisiones, mientras que las reglas jurídicas prescriben una sola. En cuanto a esta distinción ver por ejemplo HELLER, H.: La justificación del Estado, Universidad Nacional Autónoma de México (UNAM), México, 2002, p. 11. Ver también ALEXY, R.: "On the Concept and the Nature of Law", Ratio Juris, no 21(3), 2008, pp. 281-299; y DWORKIN, R.: Law's Empire, The Belknap Press of Harvard University Press, Cambridge, 1986. 
ello, imposibilitar reglas jurídicas diversas. Es eso el objeto de la siguiente parte. Trata de este reconocimiento como hecho y como obligación jurídica.

\section{La armonización de los derechos cooperativos por el reconocimiento de los principios cooperativos}

\section{La armonización de los derechos cooperativos por el reconocimiento voluntario de los principios cooperativos}

Casi todas las leyes de cooperativas regionales mencionadas integran los principios cooperativos de la $\mathrm{ACI}^{29}$. Siempre más leyes de cooperativas nacionales lo hacen también ${ }^{30}$. Las diferentes maneras de esta integración -tener en cuenta, inclusión o referencia- se clasifican de la siguiente manera: leyes que no mencionan los principios cooperativos, pero que, sí, los traducen en reglas jurídicas; leyes que se refieren a principios cooperativos; leyes que incluyen los títulos de los principios cooperativos de la ACI, a veces al modificar la terminología; leyes que no solo incluyen los títulos de los principios de la ACI, sino también las explicaciones de la ACI contenidas en la Declaración de la ACI, a veces también al modificar la terminología; leyes que incluyen además de los principios cooperativos de la ACI otros más; leyes que no los incluyen todos o que incluyen algunos y ańaden otros; leyes que mencionan el autor de los principios cooperativos (con frecuencia la $\mathrm{ACI}$ ); leyes que no mencionan el autor o que no lo hacen claramente; y, por fin, leyes que cualifican los principios cooperativos de "guía" para la interpretación de las reglas jurídicas.

Las consecuencias de estas diferentes maneras de integrar los principios cooperativos en las leyes no son claras. En todos los casos en donde las leyes modifican los principios o no revelan su autor, los principios cooperativos se transforman en principios del sistema jurídico respectivo. Su interpretación se hará de la misma manera que

29. Ver los Artículos 6 y 18, $11^{\circ}$ ) de la Ley uniforme de OHADA; el Artículo 4 de la Ley uniforme de la East African Community; el Artículo 4 de la Ley marco para las cooperativas de América Latina; y el considerando 6 del Reglamento de la Union Europea que se refiere a las Directrices de las Naciones Unidas encaminadas a crear un entorno propicio para el desarrollo de las cooperativas (Doc. A/56/73 - E/2001/68, Annex) que, a su vez, se refieren en su Punto 11 a la Declaración de la ACI.

30. Ejemplos: El Código Cooperativo Portugués (Artículo 3 : "principios de la ACI"); Ley de cooperativas del País Vasco (Artículo 1). Ver para otros ejemplos ILO: The Story..., 2015; las introducciones a los informes nacionales en CRACOGNA, D., FICI, A. \& HENRŸ, H. (eds.): International Handbook of Cooperative Law, Springer, Heidelberg, 2013; y los resultados de los "Legal Framework Analyses" de la ACI (https:/coops4dev. coop). 
la de los principios de este sistema ${ }^{31}$. Los casos en donde las leyes se refieren a la ACI como autor de los principios son más complejos. Si su interpretación se hace también según los criterios del sistema respectivo, esa interpretación puede divergir de la de la ACI. En este caso, los afiliados de la ACI e, indirectamente, los miembros de estos afiliados encuentran un dilema: la Declaración de la ACI es jurídicamente vinculante para ellos porque forma parte de los estatutos de la ACI, que es una asociación regida por el derecho privado belga.

Los casos de armonizaciones del derecho cooperativo a través de las referencias en las leyes de cooperativas a los principios cooperativos son un hecho. La cuestión es si hay una obligación jurídica que exija el reconocimiento de los principios cooperativos y su traducción en reglas jurídicas. La respuesta la encontramos en el Párrafo 10. (1) de la R. 193 de la OIT. Es el tema de la siguiente sección.

\section{La armonización obligatoria de los derechos cooperativos según el Párrafo 10. (1) de la Recomendación No. 193 de la Organización Internacional de Trabajo sobre la promoción de las cooperativas}

La Declaración de la ACI, declaración de un organismo privado, no obliga a los legisladores de reconocer los principios cooperativos de la ACI. Pero, los principios cooperativos no forman solamente parte de la Declaración de la ACI. Las Guidelines aimed at creating a supportive environment for the development of cooperatives [Directrices de las Naciones Unidas encaminadas a crear un entorno propicio para el desarrollo de las cooperativas] en $2001^{32}$ se refieren a esos principios de la ACI en su Párrafo 11 y la R. 193 de la OIT incluye principios cooperativos en su texto (Párrafo 3) y en su Anexo. La cuestión es si el Párrafo 10. (1) de la R. 193 de la OIT es jurídicamente vinculante (2.1) y cuál es el contenido de esta obligación para los legisladores respecto al derecho cooperativo (2.2). 


\subsection{El valor jurídico vinculante del Párrafo 10.(1) de la Recomendación sobre la Promoción de las Cooperativas de la Organización Internacional del Trabajo}

En cuanto al valor jurídico vinculante del Párrafo 10.(1) de la R. 193 de la OIT, los argumentos en pro se resumen así: i.) La OIT tiene competencia en materia de derecho cooperativo; ii.) la falta de efectividad del derecho público internacional no vale para negar la existencia de un derecho público internacional; iii.) si la referencia a los principios cooperativos por los legisladores se consolida, eso podrá constituir una costumbre, fuente del derecho público internacional según el Artículo 38 del Estatuto de la Corte Internacional de Justicia; iv.) la R. 193 de la OIT constituye una fuente autónoma del derecho público internacional; v.) la R. 193 de la OIT disfruta de una legitimidad democrática única, sobre todo porque integra la opinión de 700 millones de cooperativistas en forma del contenido de la Declaración de la ACI; vi.) el bajo grado de impacto de la R. 193 de la OIT sobre la soberanía de los Estados miembros de la OIT, quienes ya tienen la obligación jurídica de respetar los derechos humanos vinculantes. Según estos derechos humanos se debe permitir el establecimiento de cooperativas basadas en los principios cooperativos ${ }^{33}$; y, vii.) la Declaración de la ACI como fuente autónoma del derecho público internacional ${ }^{34}$.

\subsection{El contenido del Párrafo 10.(1) de la Recomendación sobre la Promoción de las Cooperativas de la Organización Internacional del Trabajo}

En cuanto al contenido de la obligación de los legisladores respecto a los principios cooperativos tenemos que considerar tres de los Párrafos de la R. 193 de la OIT: los Párrafos 7, 10 y 18. El Párrafo 7. (2) establece la obligación de tratar a las cooperativas según el principio del trato igual, es decir, a tener en cuenta sus especificidades determinadas por los principios cooperativos. Los Párrafos 10 y 18 se pronuncian explícitamente sobre la legislación cooperativa. Establecen lo siguiente: "10. (1) Los Estados Miembros deberían adoptar una legislación y una reglamentación específicas en materia de cooperativas, inspiradas en los valores y principios cooperativos enunciados en el párrafo 3, y revisar esta legislación y reglamentación cuando proceda." Y “18. d) La cooperación internacional debería ser facilitada mediante:... (d) el desarrollo a nivel internacional y regional de... leyes comunes de apoyo a las cooperativas, cuando proceda y sea posible...” Estos dos Párrafos plantean tres preguntas principales: ¿o

33. Ver Artículo 22 de Pacto sobre los derechos civiles y políticos en 1966.

34. Para más detalles, vid. HENRŸ, H.: "International Cooperative Law. Utopia, Realistic Utopia or Reality?", Revista Cooperativismo e Economia Social (CES), no 42, 2020, pp. 25-56. 
se contradicen estos párrafos? (2.2.1), ¿A cuáles principios cooperativos se refiere el Párrafo 10? (2.2.2), ¿Cómo traducir estos principios en reglas jurídicas? (2.2.3).

2.2.1. La relación entre los Párrafos 10. (1) y 18 de la Recomendación sobre la Promoción de las Cooperativas de la Organización Internacional del Trabajo

Prima vista, los Párrafos 10. (1) y 18. d) de la R. 193 de la OIT se contradicen. "Leyes comunes" (Párrafo 18. (d)), aún en el plural, implica uniformización. A causa de la diferencia entre principios (cooperativos) y reglas jurídicas, la formulación "legislación [... inspirada] en los... principios cooperativos" (Párrafo 10. (1)) implica diversidad. Una interpretación sistemática de los dos párrafos nos conduce a concluir a que el Párrafo 10. (1) prevalece. Eso por las siguientes razones: Varias veces el texto de la R. 193 de la OIT hace referencia al desarrollo sostenible ${ }^{35}$. Al reducir las formas de cooperativas una ley uniforme o leyes uniformes reducirían la vital diversidad, fuente de desarrollo y, por ende, fuente del desarrollo sostenible. Siempre más áreas del derecho a todos los niveles - nacional, regional e internacional - integran el concepto de desarrollo sostenible; como ya se mencionó, la Corte Internacional de Justicia lo ha reconocido, desde 1997, como concepto del derecho público internacional. Esa interpretación sistemática de los dos Párrafos 10. (1) y 18. d) de la R. 193 de la OIT no excluye unificaciones en casos excepcionales.

2.2.2. ¿A cuáles principios cooperativos se refiere el Párrafo 10. (1) de la Recomendación sobre la Promoción de las Cooperativas de la Organización Internacional del Trabajo?

La respuesta a la cuestión a cuáles principios cooperativos se refiere el Párrafo 10. (1) de la R. 193 de la OIT determina los criterios de su interpretación. Además de incluir los principios cooperativos en el Párrafo 3 y en el Anexo la R. 193 de la OIT, se refiere varias veces a principios cooperativos. Aparte de en el Párrafo 10. (1), también por ejemplo en los Párrafos 8. (1)(f) y 18. (b)(iv.). Estas referencias son ambiguas. A primera lectura, parece que la R. 193 de la OIT integra el texto de la Declaración de la ACI: la definición de las cooperativas en el Párrafo 2, los valores y los principios en el Párrafo 3 y en el Anexo de la recomendación. Pero, no es así. Primero, la R. 193 de la OIT introduce la definición de las cooperativas en el Párrafo 2 con las palabras "a los fines de esta Recomendación...". Por una parte, es una cláusula 
declaratoria general; por otra parte remite la definición en el contexto del derecho público internacional, lo que permite la mencionada interpretación sistemática de la concurrencia entre los Párrafos 10. (1) y 18. d). Segundo, el texto de la R. 193 de la OIT, a exclusión de su Anexo, no vincula los principios cooperativos a los valores cooperativos como lo hace la Declaración de la ACI al formular "Los principios de las cooperativas son pautas mediante las cuales las cooperativas llevan a la práctica sus valores." Tercero, el Párrafo 3 de la R. 193 de la OIT no diferencia entre "los valores en que se basan las cooperativas" y los "valores éticos de los socios", como lo hace la Declaración de la ACI. Esas diferencias entre la Declaración de la ACI y la R. 193 de la OIT alimenta el argumento de que la Conferencia Internacional de Trabajo quiso introducir sus propios principios cooperativos. El Párrafo 3. b) de la R. 193 de la OIT viene reforzar el argumento porque menciona como autor de los principios "el movimiento cooperativo internacional”, no la ACI. Al otro lado, el titulo del Anexo de la Recomendación "Extracto de la Declaración sobre la identidad cooperativa adoptada por la asamblea general de la Alianza Cooperativa Internacional en 1995" parece confirmar la ACI como autor de los principios mencionados en la R. 193 de la OIT. Pero, persisten dudas por varias razones: Primera, no hay consenso sobre la naturaleza jurídica de los anexos a los instrumentos internacionales. Segunda, el término "movimiento cooperativo internacional" en el Párrafo 3. b) no significa "Alianza Cooperativa Internacional". Tercera, al rechazar la propuesta de mencionar la ACI en el Párrafo 3. b) ${ }^{36}$ la Conferencia Internacional de Trabajo quiso aclarar que no reconocía - al menos no para siempre - la ACI como única representante del movimiento cooperativo internacional y confirmó las dudas en cuanto a la naturaleza jurídica de los anexos. Cuarta, el Anexo vincula, como lo hace la Declaración de la ACI, los principios a los valores cooperativos de la ACI. Si hay dudas en cuanto al autor de los principios mencionados en el Párrafo 3. b) de la R. 193 de la OIT, es que el Párrafo 3. a) de la R. 193 de la OIT, a contrario del Párrafo 3. b), no se refiere ni a la ACI ni al "movimiento cooperativo internacional" como autor de los valores. $\mathrm{Al}$ ser valores y principios de la OIT, se interpretarían según los criterios del derecho público internacional y no según los de la ACI. Eso no excluiría una toma en cuenta de la interpretación continua de su Declaración por parte de la $\mathrm{ACI}^{37}$. $\mathrm{Al}$ contrario, se la exigiría.

36. Argumento no verificado en los documentos de las sesiones de la Conferencia internacional de trabajo de los años 2001 y 2002 que trataban de la R. 193 de la OIT. Pero el autor asistió a la Conferencia en 2002 como consultor de la ACI.

37. Por ejemplo, ACI, Alianza Cooperativa Internacional, Notas de orientación para los principios cooperativos (http://ica.coop/sites/default/files/attachments/Guidance\%20Notes\%20ES.pdf) 
A pesar de esas dudas, tenemos que reconocer un consenso creciente en torno a esa cuestión: el autor de los principios cooperativos contenidos en la R. 193 de la OIT es la ACI. Pero, eso plantea cuestiones adicionales, tales como, entre otras: ¿interpretación de los principios cooperativos según los criterios de la ACI en el momento de la adopción de la R. 193 de la OIT en 2002 o según los criterios actuales de la ACI?; ¿qué pasa si la ACI modifica los principios?; ¿qué pasa si otra organización reclama la posición de representante del "movimiento cooperativo internacional"?; ¿cómo armonizar las diferencias entre la clasificación de los valores y principios por la ACI y por la OIT?

Una vez solucionadas estas cuestiones la pregunta es ¿cómo traducir los principios cooperativos en reglas jurídicas?

\subsection{3. ¿Cómo traducir los principios cooperativos en reglas y prácticas jurídicas?}

En general, valores y principios éticos y morales no confunden con reglas jurídicas. Pero, sí, las guían. Entran el mundo jurídico por la puerta de los principios jurídicos. La particularidad de los valores y principios cooperativos es que su clasificación y el uso de los términos referentes se distinguen de la filosofía y de la ciencia jurídica. Por ejemplo, los valores cooperativos de "democracia", "igualdad" y "solidaridad, mencionados en la Declaración de la ACI y en la R. 193 de la OIT, son también principios jurídicos universalmente reconocidos ${ }^{38}$. La traducción de los principios cooperativos en reglas y prácticas jurídicas requiere, pues, una serie de pasos: traducción de los valores cooperativos en principios cooperativos; adecuación de los principios cooperativos con los valores éticos y morales de la filosofía; traducción de los principios cooperativos en principios cooperativos jurídicos; traducción de esos principios cooperativos jurídicos en principios jurídicos generales; $\mathrm{y}$, traducción de los principios jurídicos en reglas y prácticas jurídicas.

38. En cuanto a los principios de democracia e igualdad, ver el Pacto sobre los derechos civiles y políticos en 1966.

En cuanto al principio de solidaridad, vid. RODOTÀ, S.: Solidarietà. Un’utopia necessaria, Laterza \& Figli, Roma-Bari, 2017; SUPIOT, A.: L’esprit de Philadelphie. La justice sociale face au marché total, Seuil, París, 2010; Ibidem: "Contribution à une analyse juridique de la crise économique de 2008", Revue internationale du Travail, no 149 (2), 2010, pp. 165 y ss.

En su Obra magistral "Les forces imaginantes du droit (4 volúmenes, Paris: Seuil 2004-2011) Mireille Delmas-Marty elabora el principio de solidaridad en sus múltiples contextos (jurídicos). 


\section{Conclusión}

Nos movemos entre la realidad de las "armonizaciones" de los derechos cooperativos y la trampa de imposibilitar el desarrollo (sostenible). ¡Reduzcamos pues las armonizaciones a lo necesario! ¡Reconozcamos los principios cooperativos y los traduzcamos en reglas y prácticas jurídicas diversas!

Quizás debemos pensar la cuestión de las armonizaciones de otra manera. Montesquieu no vivía la interculturalidad de las migraciones y de las cadenas de valor globales que vivimos hoy en día. La necesidad de armonizar resulta siempre menos de consideraciones políticas por parte de los estados nacionales o económicas por parte de actores económicos influentes, sino que la efectúan los factores de la globalización. Estos factores son la digitalización y la tele-transferibilidad de datos que, a su vez, constituyen al mismo tiempo los productos y los medios de producción esenciales. Nos conectamos más que nos relacionamos. 


\section{Bibliografía}

ALEXY, R.: "On the Concept and the Nature of Law", Ratio Juris, no 21(3), 2008, pp. 281-299. DOI: https://doi.org/10.1111/j.1467-9337.2008.00391.x

BEKHECHI, M.A.: "Quelques Notes et Réflexions sur le Statut du Droit International du Développement Durable". En: Variations sur le système international. Mélanges offerts en l'honneur du Professeur Mohamed Lamouri (dir. ESSAID, M.J.), Najah Al Jadida, Casablanca, 2010, pp. 107-137.

CARBONNIER, J.: "Le droit au non-droit". En: Paul Ricour, Jacques Ellul, Jean Carbonnier, Pierre Chaunu: Dialogues (aut. ABEL, O.), Labor et Fides, Ginebra, 2012, p. 76.

COMISIÓN DE LAS COMUNIDADES EUROPEAS [CCE]: Communication from the Commission to the Council and the European Parliament, the European Economic and Social Committee and the Committee of Regions on the promotion of co-operative societies in Europe (COM (2004) 18 final, Comisión Europea (CE), Bruselas, 2004. https://eur-lex.europa.eu/legal-content/EN/TXT/?uri=CELEX$\% 3 \mathrm{~A} 52004 \mathrm{DC} 0018$

CONSEJO ECONÓMICO Y SOCIAL [CES]: Las cooperativas en el desarrollo social (A/56/73 - E/2001/68), Consejo Económico y Social de España (CES), Madrid, 2001.

CORTE INTERNACIONAL DE JUSTICIA [CIJ]: Reports of judgments, advisory opinions and orders. Case concerning: The Gabcikovo-Nagymaros Project (Hungary/ Slovakia), Corte Internacional de Justicia (CIJ), La Haya, 1997.

CRACOGNA, D.: “The Statute of Mercosur Cooperatives". En: International Handbook of Cooperative Law (eds. CRACOGNA, D., FICI, A. \& HENRŸ, H.), Springer, Heidelberg, 2013, pp. 153-164.

DOI: https://doi.org/10.1007/978-3-642-30129-2_5

CRACOGNA, D., FICI, A. \& HENRŸ, H. (eds.): International Handbook of Cooperative Law, Springer, Heidelberg, 2013.

DELMAS-MARTY, M.: Les forces imaginantes du droit (4 vols.), Seuil, París, 20042011.

DWORKIN, R.: Law's Empire, The Belknap Press of Harvard University Press, Cambridge, 1986.

FAJARDO GARCÍA, I.G.: "El fomento de la sociedad cooperativa mediante una legislación adecuada, 40 años después”, CIRIEC-España, Revista de Economía Pública, Social y Cooperativa, no extraordinario, diciembre, 2018, pp. 141-159. 
Armonizar los derechos cooperativos. ¡Realidad! ¿necesidad? ¡trampa!

(pp. 45-66)

FISCHER, T.: "Das Statut der Europäischen Genossenschaft”. En: Genossenschaftsrecht in Europa (eds. THEURL, T. \& GREVE, R.), Shaker, Aachen, 2001, pp. 167-186.

GALLIGAN, D.J.: Law in Modern Society, Oxford University Press, Oxford, 2007. GEHNE, K.: Nachhaltige Entwicklung als Rechtsprinzip, Mohr Siebeck, Tübingen, 2011.

GOLTZBERG, S.: Le droit comparé, Presses Universitaires de France (PUF), París, 2018. DOI: https://doi.org/10.3917/puf.goltz.2018.01

HELLER, H.: La justificación del Estado, Universidad Nacional Autónoma de México (UNAM), México, 2002.

HENRŸ, H.: Orientaciones para la Legislación Cooperativa, Organización Internacional del Trabajo (OIT), Ginebra, 2013.

HENRŸ, H.: "Sustainable Development and Cooperative Law: Corporate Social Responsibility or Cooperative Social Responsibility", International and Comparative Corporate Law Journal (ICCLJ), no 10(3), 2013, pp. 58-75.

DOI: https://doi.org/10.2139/ssrn.2103047

HENRŸ, H.: “Quo Vadis Cooperative Law?”, CCIJ Report, no 72, 2014, pp. 50-61. HENRŸ, H.: "International Cooperative Law. Utopia, realistic utopia or reality?", Revista Cooperativismo e Economia Social (CES), no 42, 2020, pp. 25-56.

HIEZ, D. \& KENMOGNE SIMO, A. (eds.): Le Droit des sociétés coopératives OHA$D A$, Presses Universitaires d'Aix-Marseille Université, Marsella, 2017.

HIEZ, D. \& TADJUDJE, W.: "The OHADA Cooperative Regulation". En: International Handbook of Cooperative Law (eds. CRACOGNA, D., FICI, A. \& HENRŸ, H.), Springer, Heidelberg, 2013, pp. 89-113.

INTERNATIONAL LABOUR ORGANIZATION [ILO]: Promotion of Cooperatives Recommendation, $\mathrm{n}^{\circ}$ 193, International Labour Organization (ILO), Ginebra, 2002.

https://www.ilo.org/dyn/normlex/en/f?p=NORMLEXPU-

B:12100:0::NO::P12100_ILO_CODE:R193

INTERNATIONAL LABOUR ORGANIZATION [ILO]: The Story of the ILO's Promotion of Cooperatives Recommendation, 2002 (No. 193): A review of the process of making ILO Recommendation no. 193, its implementation and its impact, International Labour Organization (ILO), Ginebra, 2015.

http:/www.ilo.org/empent/units/cooperatives/WCMS_371631/lang--en/index. htm

KOIZUMI, T.: "Cultural Diffusion, Economic Integration and the Sovereignity of the Nation-State", Rechtstheorie. Beihefte (BH RT), no 12, 1991, pp. 313 y ss. 
LEGRAND, P.: “The Impossibility of Legal Transplants", Maastricht Journal of European and comparative Law, vol. 4(2), 1997, pp. 111-124.

DOI: https://doi.org/10.1177/1023263X9700400202

De LUCA, N.: "Russia". En: International Handbook of Cooperative Law (eds. CRACOGNA, D., FICI, A. \& HENRŸ, H.), Springer, Heidelberg, 2013, pp. 667685. DOI: https://doi.org/10.1007/978-3-642-30129-2

MATTILA, H.E.S.: El latín jurídico. Historia, uso internacional, problemas de comunicación, Ediciones Jurídicas Olejnik, Santiago de Chile, 2020.

MIRIBUNG, G.: The Agricultural Cooperative in the Framework of the European Cooperative Society. Discussing and Comparing Issues of Cooperative Governance and Finance in Italy and Austria, Springer, Cham, 2020.

MORENO FONTELA, J.L.: "Las relaciones entre los valores y principios cooperativos y los principios de la normativa cooperativa", REVESCO. Revista de Estudios Cooperativos, no 123, 2017, pp. 114-127.

DOI: https://doi.org/10.5209/REVE.54923

MÜNKNER, H.H. (ed.): One Hundred Years: Co-operative Credit Societies Act in India - A Unique Experience of Legal Social Engineering, Centenary Round Table on Indian Co-operative Movement - Retrospect and Prospect, Marburg, 2005. RODOTÀ, S.: Solidarietà. Un'utopia necessaria, Laterza \& Figli, Roma-Bari, 2017.

SCHULZE, R. (ed.): Europäische Genossenschaft SCE - Handbuch, Nomos, Baden, 2004.

SUPIOT, A.: "Contribution à une analyse juridique de la crise économique de 2008”, Revue internationale du Travail, no 149(2), 2010, pp. 165-176.

SUPIOT, A.: L'esprit de Philadelphie. La justice sociale face au marché total, Seuil, París, 2010.

TADJUDJE, W.: "The East African Community's Cooperative Regulation”, International Journal of Cooperative Law (IJCL), no 1, 2018, pp. 148-166.

TADJUDJE, W.: "Standarization of cooperative law in Africa: A comparative analysis between the Ohada Uniform Act related to cooperative societies and the East Africa community's co-operative societies bill", International Journal of Cooperative Law (IJCL), no 3, 2020, pp. 31-45.

TADJUDJE, W. (dir.): Le droit des cooperatives en Afrique. Réflexions sur l'Acte uniforme de l'OHADA, ÉPURE - Éditions et Presses universitaires de Reims, Reims, 2021. VILLAFÁNEZZ PÉREZ, I.: "Algunas reflexiones en torno a la necesidad de integrar la perspectiva cooperativa en el estudio y desarrollo del ordenamiento jurídico". En: Co-operative Studies in Education Curricula. New Forms of Learning and Teaching (eds. HENRŸ, H., HYTINKOSKI, P. \& KLÉN, T.), Ruralia Institute of the University of Helsinki, Seinäjoki, 2017, pp. 54-71. 